\title{
A priori bounds of the solution of a one point IBVP for a singular fractional evolution equation
}

\author{
Said Mesloub ${ }^{1 *}$ and Hassan Eltayeb Gadain ${ }^{1}$
}

\section{"Correspondence:}

mesloub@ksu.edu.sa

${ }^{1}$ Mathematics Department, College of Science, King Saud University,

P.O. Box 2455, Riyadh, Saudi Arabia

\begin{abstract}
A priori bounds constitute a crucial and powerful tool in the investigation of initial boundary value problems for linear and nonlinear fractional and integer order differential equations in bounded domains. We present herein a collection of a priori estimates of the solution for an initial boundary value problem for a singular fractional evolution equation (generalized time-fractional wave equation) with mass absorption. The Riemann-Liouville derivative is employed. Results of uniqueness and dependence of the solution upon the data were obtained in two cases, the damped and the undamped case. The uniqueness and continuous dependence (stability of solution) of the solution follows from the obtained a priori estimates in fractional Sobolev spaces. These spaces give what are called weak solutions to our partial differential equations (they are based on the notion of the weak derivatives). The method of energy inequalities is used to obtain different a priori estimates.
\end{abstract}

Keywords: Fractional wave equation; Damping term; Energy estimate; Uniqueness of solution; Mass absorption; One point boundary value problem

\section{Introduction}

In the disk, $D=\left\{(x, y) \in \mathbb{R}^{2}, x^{2}+y^{2}<b^{2}\right\}$, we consider the following evolution fractional partial differential equation in the Riemann-Liouville sense:

$$
\partial_{t}^{\lambda} \mathcal{U}-\Delta \mathcal{U}+\delta \mathcal{U}_{t}+A(x, y, t) \mathcal{U}=h(x, y, t), \quad \forall(x, y) \in D, t>0
$$

with a damping effect due to the term $\delta \mathcal{U}_{t}$, with $\delta>0$, and where $\partial_{t}^{\lambda} \mathcal{U}$ is the RiemannLiouville fractional derivative of order $\lambda \in(1,2]$ defined below. The functions $h(x, y, t)$ and $A(x, y, t)$ are given and will be specified later. If we search only for the radially symmetric solution (the functions $h$ and $A$ must be radial), we then have the partial differential equation

$$
\partial_{t}^{\lambda} \mathcal{U}-\frac{1}{r}\left(r \mathcal{U}_{r}\right)_{r}+\delta \mathcal{U}_{t}+A(r, \theta, t) \mathcal{U}(r, t)=h(r, \theta, t),
$$

such that $0<r<b, 0<\theta<2 \pi, t>0$.

(c) The Author(s) 2020. This article is licensed under a Creative Commons Attribution 4.0 International License, which permits use sharing, adaptation, distribution and reproduction in any medium or format, as long as you give appropriate credit to the original author(s) and the source, provide a link to the Creative Commons licence, and indicate if changes were made. The images or other third party material in this article are included in the article's Creative Commons licence, unless indicated otherwise in a credit line to the material. If material is not included in the article's Creative Commons licence and your intended use is not permitted by statutory regulation or exceeds the permitted use, you will need to obtain permission directly from the copyright holder. To view a copy of this licence, visit http://creativecommons.org/licenses/by/4.0/. 
Assume that the source term $h(r, \theta, t)$ and the function $A(r, \theta, t)$ are independent of $\theta$ and denote $x$ in place of $r$, then instead of Eq. (1.2), we obtain the time-fractional generalized wave equation

$$
\partial_{t}^{\lambda} \mathcal{U}-\frac{1}{x}\left(x \mathcal{U}_{x}\right)_{x}+\delta \mathcal{U}_{t}+A(x, t) \mathcal{U}(x, t)=h(x, t),
$$

where $x \in \Omega=(0, b), t>0$.

If $A(x, t)=0$, then (1.3) reduces to the fractional non-homogeneous damped wave equation with Bessel operator

$$
\partial_{t}^{\lambda} \mathcal{U}-\frac{1}{x}\left(x \mathcal{U}_{x}\right)_{x}+\delta \mathcal{U}_{t}=h(x, t)
$$

In the special case where $\delta=0$ (no damping) and $A(x, t)=\alpha$, the differential equation (1.3) corresponds to the time-fractional generalization of the nonhomogeneous Klein-Gordon equation [1-4] with Bessel operator

$$
\partial_{t}^{\lambda} \mathcal{U}-\frac{1}{x}\left(x \mathcal{U}_{x}\right)_{x}+\alpha \mathcal{U}(x, t)=h(x, t)
$$

This equation plays a great role in relativistic physics and can be used to represent dispersive wave phenomena. In Eq. (1.5), the case $\alpha>0$ corresponds to mass absorption and the case $\alpha<0$ corresponds to mass release. When $\lambda=2, A(x, t)=0$, and $\delta>0$, Eq. (1.3) represents the telegraph equation with Bessel operator, which arises for example in signal analysis [5], wave propagation [6], and it also governs the electrical transmission in a telegraph cable,

$$
\mathcal{U}_{t t}-\frac{1}{x}\left(x \mathcal{U}_{x}\right)_{x}+\delta \mathcal{U}_{t}=h(x, t), \quad x \in(0, b), t \in[0, T]
$$

It can also be considered as the damping wave equation with damping term $\delta \mathcal{U}_{t}$. It should be noted that when $\lambda=2$, Eq. (1.5) reduces to the non-homogeneous hyperbolic KleinGordon equation with Bessel operator

$$
\mathcal{U}_{t t}-\frac{1}{x}\left(x \mathcal{U}_{x}\right)_{x}+\alpha \mathcal{U}(x, t)=h(x, t)
$$

which can be encountered for example in quantum and classical mechanics and solid state physics [6, 10]. Equation (1.5) is in fact the time-fractional counterpart of Eq. (1.7). It should be also noted that when $\lambda=1, \alpha>0$, Eq. (1.5) represents the classical parabolic diffusion equation with heat or mass absorption and source heat $h(x, t)$ where the elliptic part is replaced by the Bessel operator $\frac{1}{x} \frac{\partial}{\partial x}\left(x \frac{\partial}{\partial x}\right)$, that is,

$$
\mathcal{U}_{t}-\frac{1}{x}\left(x \mathcal{U}_{x}\right)_{x}+\alpha \mathcal{U}(x, t)=h(x, t)
$$

We also mention the very special case of Eq. (1.3), when $A=0, \delta=0$; we then have the time-fractional diffusion-wave equation for $\lambda \in(0,2]$

$$
\partial_{t}^{\lambda} \mathcal{U}-\frac{1}{x}\left(x \mathcal{U}_{x}\right)_{x}=h(x, t)
$$


where the case $\lambda \in(0,1)$ corresponds to the slow diffusion, with heat supply $h(x, t)$, and the case $\lambda \in(1,2]$ corresponds to the fast diffusion (fractional wave propagation) with $h(x, t)$ as an external force. The case $\lambda=0$ is known as the localized diffusion and corresponds to the Helmholtz equation, and the case $\lambda=2$ is known as the ballistic diffusion and corresponds to the wave equation. The differential equation (1.9) describes several physical phenomena in many branches of sciences [7-9].

Finally, we indicate that, if in Eq. (1.3) the function $\mathcal{U}$ depends only on time $t$ and $\lambda=2$, we obtain the general Bagley-Torvik equation,

$$
\mathcal{U}_{t t}+\delta \partial_{t}^{\beta} \mathcal{U}+A \mathcal{U}=h(t)=h(t), \quad t \in[0, T], \beta \in(1,2)
$$

but with $\beta=1$. This fractional differential equation has a huge applications in engineering and applied sciences fields (see for example [10-13]). There are some recent papers dealing with numerical and theoretical aspects of fractional partial differential equations, related to our work, and having many applications, for which the reader can refer to [14-21].

The main purpose of this paper is to derive certain a priori estimates (in the form of uniform bounds for derivatives) for solutions of an initial boundary value problem for a singular time-fractional wave equation with mass absorption in the case of presence and absence of a certain damping term. The method of energy inequalities is used to obtain different a priori estimates (see for example [22-24]). The obtained results can greatly contribute in the development of the method of a priori estimate, the so called energy inequalities method for fractional order PDEs. To the best of our knowledge, there are few papers dealing with the a priori estimate method for the fractional order differential equations case.

\section{Statement of the problem}

A time-fractional damped partial differential equation with Bessel operator in the domain $\Lambda=\Omega \times[0, T]=(0, b) \times[0, T]$ is considered

$$
\partial_{t}^{\lambda} \mathcal{U}-\frac{1}{x}\left(x \mathcal{U}_{x}\right)_{x}+\delta \mathcal{U}_{t}+A(x, t) \mathcal{U}(x, t)=h(x, t),
$$

under zero initial data

$$
\mathcal{U}(x, 0)=0, \quad \mathcal{U}_{t}(x, 0)=0, \quad x \in(0, b)
$$

and with one point boundary condition (Dirichlet condition)

$$
\mathcal{U}(b, t)=0, \quad t \in[0, T]
$$

where $h \in L_{x}^{2}([0, T], \Omega)$ and the function $A$ satisfies

$$
A(x, t) \geq A_{0}>0, \quad 0<A_{t}(x, t) \leq A_{1}, \quad \forall(x, t) \in \Lambda .
$$

The fractional order derivative $\partial_{t}^{\lambda} \mathcal{Z}$ is the left Riemann-Liouville fractional derivative of order $\lambda \in(1,2]$ for a function $\mathcal{Z}$ defined on $\Lambda[25,26]$ given by

$$
\partial_{t}^{\eta} \mathcal{Z}(x, t)=\frac{1}{\Gamma(1-\eta)} \frac{d}{d t} \int_{0}^{t} \frac{\mathcal{Z}(x, s)}{(t-s)^{\eta}} d s, \quad t>0 .
$$


We denote by $\partial_{T}^{\eta} \mathcal{Z}$ the right Riemann-Liouville fractional derivative defined by

$$
\partial_{T}^{\eta} \mathcal{Z}(x, t)=-\frac{1}{\Gamma(1-\eta)} \frac{d}{d t} \int_{t}^{T} \frac{\mathcal{Z}(x, s)}{(s-t)^{\eta}} d s, \quad t<T
$$

For essentials of fractional calculus, we refer the reader to [25-28].

\section{Functional setting}

Let $L^{2}(0, T)$ be the space of measurable square integrable functions on $(0, T)$ with inner product and norm given, respectively, by

$$
(U, V)_{L^{2}(0, T)}=\int_{0}^{T} U V d t, \quad\|U\|_{L^{2}(0, T)}^{2}=\int_{0}^{T} U^{2} d t
$$

and $L_{x}^{2}(0, b)$, be the weighted space having the inner product

$$
(U, V)_{L_{x}^{2}(0, b)}=\int_{0}^{b} x U V d x
$$

We also introduce the fractional derivative spaces $H_{t, x}^{\eta}([0, T], \Omega)$ and $H_{T, x}^{\eta}([0, T], \Omega)$ to be the space of functions $U \in L_{x}^{2}([0, T], \Omega)$ having $\eta$-order Riemann-Liouville derivative $\partial_{t}^{\eta} U \in L_{x}^{2}([0, T], \Omega), U(x, 0)=0, U(x, T)=0$, having, respectively, the norms

$$
\|U\|_{H_{t, x}^{\eta}([0, T], \Omega)}^{2}=\|U\|_{L_{x}^{2}([0, T], \Omega)}^{2}+\left\|\partial_{t}^{\eta} U\right\|_{L_{x}^{2}([0, T], \Omega)}^{2}
$$

and

$$
\|U\|_{H_{T, x}^{\eta}([0, T], \Omega)}^{2}=\|U\|_{L_{x}^{2}([0, T], \Omega)}^{2}+\left\|\partial_{T}^{\eta} U\right\|_{L_{x}^{2}([0, T], \Omega)}^{2} .
$$

That is, $H_{t, x}^{\eta}([0, T], \Omega)$ and $H_{T, x}^{\eta}([0, T], \Omega)$ can be defined as the closure of $C^{\infty}([0, T], \Omega)$ with respect to the norms (3.3) and (3.4), respectively. We denote by $C([0, T], \Omega)$ the Banach space of the set of functions $U:[0, T] \rightarrow \Omega$ equipped with the norm

$$
\|U\|_{C([0, T], \Omega)}=\max _{0 \leq t \leq T}\|U(\cdot, t)\|_{\Omega} .
$$

We also need the usual Sobolev space $H_{x}^{1}(\Lambda)$ with norm

$$
\|\mathcal{U}\|_{H_{x}^{1}(\Lambda)}^{2}=\|\mathcal{U}\|_{L_{x}^{2}(\Lambda)}^{2}+\left\|\mathcal{U}_{x}\right\|_{L_{x}^{2}(\Lambda)}^{2} .
$$

The following lemmas are crucial for the proofs of results.

Lemma 1 ([29]) If $0<\eta<1, \mathcal{N}(t) \in H_{t}^{\eta}([0, T]), \mathcal{M}(t) \in H_{T}^{\eta}([0, T])$, and $\mathcal{N}(0)=0, \mathcal{M}(0)=$ 0. then

$$
\left(\partial_{t}^{\eta} \mathcal{N}, \mathcal{M}\right)_{L^{2}([0, T]}=\left(\mathcal{N}, \partial_{T}^{\eta} \mathcal{M}\right)_{L^{2}([0, T])} .
$$


Lemma 2 ([30]) If $\beta>0, \mathcal{N} \in C^{\infty}(\mathbb{R})$ and $\operatorname{supp} \mathcal{N} \subset[0, T]$. Then

$$
\left(\partial_{t}^{\beta} \mathcal{N}, \partial_{T}^{\beta} \mathcal{N}\right)_{L^{2}([0, T])}=\cos (\pi \beta)\left\|\partial_{t}^{\beta} \mathcal{N}\right\|_{L^{2}(0, \infty)}^{2} .
$$

Remark (see [30], pages 5 and 19) Note that, for $\beta=n-\frac{1}{2}, n$ is a natural number, $\left(\partial_{t}^{\beta} \mathcal{N}, \partial_{T}^{\beta} \mathcal{N}\right)_{L^{2}([0, T])}=0$. For example $\beta=\frac{1}{2},\left(\partial_{t}^{\frac{1}{2}} \mathcal{N}, \partial_{T}^{\frac{1}{2}} \mathcal{N}\right)_{L^{2}([0, T])}=\left(\partial_{t} \mathcal{N}, \mathcal{N}\right)_{L^{2}([0, T])}=0$, provided that $\mathcal{N}$ vanishes on the boundary.

Throughout the following, we suppose that there exists a solution $u$ in $C^{2,2}(\bar{\Lambda})$ of problem (2.1)-(2.3) and problem (5.1), (2.2) and (2.3).

\section{A priori estimations for the damped case}

Theorem 4.1 Let $\lambda=\eta+1$ with $0<\eta<1$, and assume that $h \in L_{x}^{2}([0, T], \Omega)$, and the function $A(x, t)$ satisfies conditions (2.4), then the solution of problem (2.1)-(2.3) verifies the a priori bound

$$
\begin{aligned}
& \|\mathcal{U}\|_{C\left(0, T ; H_{t, x}^{\eta}(\Omega)\right)}^{2}+\left\|\mathcal{U}_{t}\right\|_{H_{t, x}^{\eta / 2}(\Lambda)}^{2}+\left\|\partial_{t}^{\eta / 2} \mathcal{U}\right\|_{H_{x}^{1}(\Lambda)}^{2} \\
& \quad \leq \omega e^{\omega T}\|h\|_{L_{x}^{2}(\Lambda)}^{2}
\end{aligned}
$$

where $\omega$ is a positive constant given by

$$
\omega=\frac{\max \left(\frac{1}{2}+\delta, \frac{1}{2}+\frac{1}{\delta}, A_{1}\right)}{\min \left(1, \frac{\delta}{2}, 2 \cos (\pi \eta / 2), 2 A_{0} \cos (\pi \eta / 2)\right.} .
$$

Proof We consider the inner product in $L_{x}^{2}(\Omega)$ of the differential equation (2.1) and the operator $P(\mathcal{U})=\left(\partial_{t}^{\eta}+\frac{\partial}{\partial t}\right) \mathcal{U}$ to have

$$
\begin{aligned}
\left(\partial_{t}^{\eta+1}\right. & \left.\left.\mathcal{U}, \partial_{t}^{\eta} \mathcal{U}\right)_{L_{x}^{2}(\Omega)}-\left(\left(x \mathcal{U}_{x}\right)_{x}, \partial_{t}^{\eta} \mathcal{U}\right)\right)_{L^{2}(\Omega)}+\left(\delta \mathcal{U}_{t}, \partial_{t}^{\eta} \mathcal{U}\right)_{L_{x}^{2}(\Omega)} \\
& \left.+\left(\partial_{t}^{\eta+1} \mathcal{U}, \mathcal{U}_{t}\right)_{L_{x}^{2}(\Omega)}-\left(\left(x \mathcal{U}_{x}\right)_{x}, \mathcal{U}_{t}\right)\right)_{L^{2}(\Omega)}+\left(\delta \mathcal{U}_{t}, \mathcal{U}_{t}\right)_{L_{x}^{2}(\Omega)} \\
\quad & \times\left(A \mathcal{U}, \partial_{t}^{\eta} \mathcal{U}\right)_{L_{x}^{2}(\Omega)}+\left(A \mathcal{U}, \mathcal{U}_{t}\right)_{L_{x}^{2}(\Omega)} \\
= & \left(h, \partial_{t}^{\eta} \mathcal{U}\right)_{L_{x}^{2}(\Omega)}+\left(h, \mathcal{U}_{t}\right)_{L_{x}^{2}(\Omega)} .
\end{aligned}
$$

Taking into account the boundary condition (2.3) and the initial conditions (2.2), we obtain

$$
\begin{aligned}
& \left(\partial_{t}^{\eta+1} \mathcal{U}, \partial_{t}^{\eta} \mathcal{U}\right)_{L_{x}^{2}(\Omega)}=\frac{1}{2} \frac{\partial}{\partial t}\left\|\partial_{t}^{\eta} \mathcal{U}\right\|_{L_{x}^{2}(\Omega)}^{2} \\
& \left.-\left(\left(x \mathcal{U}_{x}\right)_{x}, \partial_{t}^{\eta} \mathcal{U}\right)\right)_{L^{2}(\Omega)}=\left(\partial_{t}^{\eta} \mathcal{U}_{x}, \mathcal{U}_{x}\right)_{L_{x}^{2}(\Omega)} \\
& \left(\partial_{t}^{\eta+1} \mathcal{U}, \mathcal{U}_{t}\right)_{L_{x}^{2}(\Omega)}=\left(\partial_{t}^{\eta} \mathcal{U}_{t}, \mathcal{U}_{t}\right)_{L_{x}^{2}(\Omega)} \\
& \left.-\left(\left(x \mathcal{U}_{x}\right)_{x}, \mathcal{U}_{t}\right)\right)_{L^{2}(\Omega)}=\frac{1}{2} \frac{\partial}{\partial t}\left\|\mathcal{U}_{x}\right\|_{L_{x}^{2}(\Omega)}^{2} \\
& \left(A \mathcal{U}, \mathcal{U}_{t}\right)_{L_{x}^{2}(\Omega)}=\frac{1}{2} \frac{\partial}{\partial t}\|\sqrt{A} \mathcal{U}\|_{L_{x}^{2}(\Omega)}^{2}-\frac{1}{2}\left\|\sqrt{A_{t}} \mathcal{U}\right\|_{L_{x}^{2}(\Omega)}^{2}
\end{aligned}
$$


Referring to (4.3) and using (4.4)-(4.8), it follows that

$$
\begin{aligned}
\frac{\partial}{\partial t} \| & \partial_{t}^{\eta} \mathcal{U}\left\|_{L_{x}^{2}(\Omega)}^{2}+\frac{\partial}{\partial t}\right\| \mathcal{U}_{x} \|_{L_{x}^{2}(\Omega)}^{2}+2\left(\partial_{t}^{\eta} \mathcal{U}_{t}, \mathcal{U}_{t}\right)_{L_{x}^{2}(\Omega)} \\
& +2\left(\partial_{t}^{\eta} \mathcal{U}_{x}, \mathcal{U}_{x}\right)_{L_{x}^{2}(\Omega)}+2 \delta\left\|\mathcal{U}_{t}\right\|_{L_{x}^{2}(\Omega)}^{2}+\left(A \mathcal{U}, \partial_{t}^{\eta} \mathcal{U}\right)_{L_{x}^{2}(\Omega)} \\
& +\frac{1}{2} \frac{\partial}{\partial t}\|\sqrt{A} \mathcal{U}\|_{L_{x}^{2}(\Omega)}^{2} \\
= & 2\left(h, \partial_{t}^{\eta} \mathcal{U}\right)_{L_{x}^{2}(\Omega)}+2\left(h, \mathcal{U}_{t}\right)_{L_{x}^{2}(\Omega)}-2\left(\delta \mathcal{U}_{t}, \partial_{t}^{\eta} \mathcal{U}\right)_{L_{x}^{2}(\Omega)} \\
& +\left\|\sqrt{A_{t}} \mathcal{U}\right\|_{L_{x}^{2}(\Omega)}^{2} .
\end{aligned}
$$

Replace $t$ by $s$ and integrate with respect to $s$ over the interval $(0, t)$ and use Lemma 1 and Lemma 2 and conditions (2.4); it follows that

$$
\begin{aligned}
& \left\|\partial_{t}^{\eta} \mathcal{U}(\cdot, t)\right\|_{L_{x}^{2}(\Omega)}^{2}+2 \cos \left(\frac{\pi \eta}{2}\right)\left\|\partial_{t}^{\eta / 2} \mathcal{U}_{x}\right\|_{L^{2}\left(0, \infty ; L_{x}^{2}(\Omega)\right)}^{2}+\left\|\mathcal{U}_{x}(\cdot t)\right\|_{L_{x}^{2}(\Omega)}^{2} \\
& \quad+\delta\left\|\mathcal{U}_{s}\right\|_{L^{2}\left(0, t ; L_{x}^{2}(\Omega)\right)}^{2}+2 \cos \left(\frac{\pi \eta}{2}\right)\left\|\partial_{t}^{\eta / 2} \mathcal{U}_{t}\right\|_{L^{2}\left(0, \infty ; L_{x}^{2}(\Omega)\right)}^{2} \\
& \quad+A_{0}\|\mathcal{U}(\cdot, t)\|_{L_{x}^{2}(\Omega)}^{2}+2 A_{0} \cos \left(\frac{\pi \eta}{2}\right)\left\|\partial_{t}^{\eta / 2} \mathcal{U}\right\|_{L^{2}\left(0, \infty ; L_{x}^{2}(\Omega)\right)}^{2} \\
& \leq \int_{0}^{t}\left(h, \partial_{s}^{\eta} \mathcal{U}\right)_{L_{x}^{2}(\Omega)} d s-\delta \int_{0}^{t}\left(\partial_{s}^{\eta} \mathcal{U}, \mathcal{U}_{s}\right)_{L_{x}^{2}(\Omega)} d s \\
& \quad+\int_{0}^{t}\left(h, \mathcal{U}_{s}\right)_{L_{x}^{2}(\Omega)} d s+A_{1} \int_{0}^{t}\|\mathcal{U}(\cdot, s)\|_{L_{x}^{2}(\Omega)}^{2} d s .
\end{aligned}
$$

Since $\cos \left(\frac{\pi \eta}{2}\right)>0$ for $\eta \in(0,1)$, and

$$
\begin{aligned}
& \left\|\partial_{t}^{\eta / 2} \mathcal{U}_{x}\right\|_{L^{2}\left(0, \infty ; L_{x}^{2}(\Omega)\right)}^{2} \geq\left\|\partial_{t}^{\eta / 2} \mathcal{U}_{x}\right\|_{L^{2}\left(0, t ; L_{x}^{2}(\Omega)\right)}^{2}, \\
& \left\|\partial_{t}^{\eta / 2} \mathcal{U}_{t}\right\|_{L^{2}\left(0, \infty ; L_{x}^{2}(\Omega)\right)}^{2} \geq\left\|\partial_{t}^{\eta / 2} \mathcal{U}_{t}\right\|_{L^{2}\left(0, t ; L_{x}^{2}(\Omega)\right)}^{2},
\end{aligned}
$$

inequality (4.10) becomes

$$
\begin{aligned}
& \left\|\partial_{t}^{\eta} \mathcal{U}(\cdot, t)\right\|_{L_{x}^{2}(\Omega)}^{2}+2 \cos \left(\frac{\pi \eta}{2}\right)\left\|\partial_{t}^{\eta / 2} \mathcal{U}_{x}\right\|_{L_{x}^{2}\left(\Lambda_{t}\right)}^{2}+\left\|\mathcal{U}_{x}(\cdot t)\right\|_{L_{x}^{2}(\Omega)}^{2} \\
& \quad+\delta\left\|\mathcal{U}_{t}\right\|_{L_{x}^{2}\left(\Lambda_{t}\right)}^{2}+2 \cos \left(\frac{\pi \eta}{2}\right)\left\|\partial_{t}^{\eta / 2} \mathcal{U}_{t}\right\|_{L_{x}^{2}\left(\Lambda_{t}\right)}^{2} \\
& \quad \times 2 A_{0} \cos \left(\frac{\pi \eta}{2}\right)\left\|\partial_{t}^{\eta / 2} \mathcal{U}\right\|_{L_{x}^{2}\left(\Lambda_{t}\right)}^{2}+A_{0}\|\mathcal{U}(\cdot, t)\|_{L_{x}^{2}(\Omega)}^{2} \\
& \leq\left(h, \partial_{t}^{\eta} \mathcal{U}\right)_{L_{x}^{2}\left(\Lambda_{t}\right)}-\delta\left(\partial_{t}^{\eta} \mathcal{U}, \mathcal{U}_{t}\right)_{L_{x}^{2}\left(\Lambda_{t}\right)}+\left(h, \mathcal{U}_{t}\right)_{L_{x}^{2}\left(\Lambda_{t}\right)} \\
& \quad+A_{1}\|\mathcal{U}\|_{L_{x}^{2}\left(\Lambda_{t}\right)}^{2}
\end{aligned}
$$


where $\Lambda_{t}=\Omega \times(0, t)$. We estimate the right-hand side of (4.11) which becomes

$$
\begin{aligned}
& \left\|\partial_{t}^{\eta} \mathcal{U}(\cdot, t)\right\|_{L_{x}^{2}(\Omega)}^{2}+2 \cos \left(\frac{\pi \eta}{2}\right)\left\|\partial_{t}^{\eta / 2} \mathcal{U}_{x}\right\|_{L_{x}^{2}\left(\Lambda_{t}\right)}^{2}+\left\|\mathcal{U}_{x}(\cdot t)\right\|_{L_{x}^{2}(\Omega)}^{2} \\
& +\delta\left\|\mathcal{U}_{t}\right\|_{L_{x}^{2}\left(\Lambda_{t}\right)}^{2}+2 \cos \left(\frac{\pi \eta}{2}\right)\left\|\partial_{t}^{\eta / 2} \mathcal{U}_{t}\right\|_{L_{x}^{2}\left(\Lambda_{t}\right)}^{2} \\
& \quad+2 A_{0} \cos \left(\frac{\pi \eta}{2}\right)\left\|\partial_{t}^{\eta / 2} \mathcal{U}\right\|_{L_{x}^{2}\left(\Lambda_{t}\right)}^{2}+A_{0}\|\mathcal{U}(\cdot, t)\|_{L_{x}^{2}(\Omega)}^{2} \\
& \leq \frac{\varepsilon_{1}}{2}\left\|\partial_{t}^{\eta} \mathcal{U}\right\|_{L_{x}^{2}\left(\Lambda_{t}\right)}^{2}+\frac{1}{2 \varepsilon_{1}}\|h\|_{L_{x}^{2}\left(\Lambda_{t}\right)}^{2}+\frac{\delta \varepsilon_{2}}{2}\left\|\mathcal{U}_{t}\right\|_{L_{x}^{2}\left(\Lambda_{t}\right)}^{2}+A_{1}\|\mathcal{U}\|_{L_{x}^{2}\left(\Lambda_{t}\right)}^{2} \\
& +\frac{\delta}{2 \varepsilon_{2}}\left\|\partial_{t}^{\eta} \mathcal{U}\right\|_{L_{x}^{2}\left(\Lambda_{t}\right)}^{2}+\frac{\varepsilon_{3}}{2}\left\|\mathcal{U}_{t}\right\|_{L_{x}^{2}\left(\Lambda_{t}\right)}^{2}+\frac{1}{2 \varepsilon_{3}}\|h\|_{L_{x}^{2}\left(\Lambda_{t}\right)}^{2}
\end{aligned}
$$

Let $\varepsilon_{1}=1, \varepsilon_{2}=\frac{1}{2}, \varepsilon_{3}=\frac{\delta}{2}$, then (4.12) reduces to

$$
\begin{aligned}
& \left\|\partial_{t}^{\eta} \mathcal{U}(\cdot, t)\right\|_{L_{x}^{2}(\Omega)}^{2}+\|\mathcal{U}(\cdot, t)\|_{L_{x}^{2}(\Omega)}^{2}+\left\|\mathcal{U}_{x}(\cdot t)\right\|_{L_{x}^{2}(\Omega)}^{2} \\
& \quad+\left\|\mathcal{U}_{t}\right\|_{L_{x}^{2}\left(\Lambda_{t}\right)}^{2}+\left\|\partial_{t}^{\eta / 2} \mathcal{U}_{t}\right\|_{L_{x}^{2}\left(\Lambda_{t}\right)}^{2}+\left\|\partial_{t}^{\eta / 2} \mathcal{U}\right\|_{L_{x}^{2}\left(\Lambda_{t}\right)}^{2} \\
& \quad+\left\|\partial_{t}^{\eta / 2} \mathcal{U}_{x}\right\|_{L_{x}^{2}\left(\Lambda_{t}\right)}^{2} \\
& \leq \omega\left(\left\|\partial_{t}^{\eta} \mathcal{U}\right\|_{L_{x}^{2}\left(\Lambda_{t}\right)}^{2}+\|\mathcal{U}\|_{L_{x}^{2}\left(\Lambda_{t}\right)}^{2}+\|h\|_{L_{x}^{2}\left(\Lambda_{t}\right)}^{2}\right),
\end{aligned}
$$

where

$$
\omega=\frac{\max \left(\frac{1}{2}+\delta, \frac{1}{2}+\frac{1}{\delta}, A_{1}\right)}{\min \left(1, \frac{\delta}{2}, 2 \cos (\pi \eta / 2), 2 A_{0} \cos (\pi \eta / 2)\right.} .
$$

We apply Gronwall's lemma [11] to (4.13) and we omit the third term on its left-hand side, and we get

$$
\begin{aligned}
& \left\|\partial_{t}^{\eta} \mathcal{U}(\cdot, t)\right\|_{L_{x}^{2}(\Omega)}^{2}+\|\mathcal{U}(\cdot, t)\|_{L_{x}^{2}(\Omega)}^{2} \\
& \quad+\left\|\partial_{t}^{\eta / 2} \mathcal{U}_{x}\right\|_{L_{x}^{2}\left(\Lambda_{t}\right)}^{2}+\left\|\partial_{t}^{\eta / 2} \mathcal{U}\right\|_{L_{x}^{2}\left(\Lambda_{t}\right)}^{2} \\
& \quad+\left\|\mathcal{U}_{t}\right\|_{L_{x}^{2}\left(\Lambda_{t}\right)}^{2}+\left\|\partial_{t}^{\eta / 2} \mathcal{U}_{t}\right\|_{L_{x}^{2}\left(\Lambda_{t}\right)}^{2} \\
& \leq \omega e^{\omega t}\|h\|_{L_{x}^{2}\left(\Lambda_{t}\right)}^{2}
\end{aligned}
$$

By taking the maximum of both sides of (4.15) with respect to $t$ over $[0, T]$, then it can be reduced to the desired a priori estimate

$$
\begin{aligned}
& \|\mathcal{U}\|_{C\left(0, T ; H_{t, x}^{\eta}(\Omega)\right)}^{2}+\left\|\mathcal{U}_{t}\right\|_{H_{t, x}^{\eta / 2}(\Lambda)}^{2}+\left\|\partial_{t}^{\eta / 2} \mathcal{U}\right\|_{H_{x}^{1}(\Lambda)}^{2} \\
& \quad \leq \omega e^{\omega T}\|h\|_{L_{x}^{2}(\Lambda)}^{2}
\end{aligned}
$$

where $\omega$ is given by (4.14). It follows from the a priori estimate (4.16) that problem (2.1)(2.3) admits a unique solution that depends continuously on the given data. 


\section{A priori estimations for the undamped case}

In the case of the undamped equation

$$
\partial_{t}^{\lambda} \mathcal{U}-\frac{1}{x}\left(x \mathcal{U}_{x}\right)_{x}+A(x, t) \mathcal{U}(x, t)=h(x, t),
$$

that is, when $\delta=0$, we have the following results.

Theorem 5.1 Suppose that $A(x, t)$ satisfies conditions (2.4) and $h \in L_{x}^{2}([0, T], \Omega)$. Let $\lambda=$ $\eta+1$ with $0<\eta<1$, then the solution of problem (5.1), (2.2) and (2.3) satisfies the a priori estimate

$$
\max _{0 \leq \tau \leq T}\|\mathcal{U}(x \cdot \tau)\|_{H_{x}^{1}(\Omega)}^{2}+\left\|\partial_{t}^{\eta / 2} \mathcal{U}_{t}\right\|_{L_{x}^{2}(\Lambda)}^{2} \leq \theta \exp (\theta T)\|h\|_{L_{x}^{2}(\Lambda)}^{2}
$$

where

$$
\theta=\frac{\max \left(\frac{T^{\eta / 2}}{\cos \left(\frac{\pi \eta}{2}\right) \cdot \Gamma\left(\frac{\eta}{2}+1\right)}, A_{1}\right)}{\min \left(\cos \left(\frac{\pi \eta}{2}\right), 1\right)} .
$$

Proof Consider the identity

$$
\begin{aligned}
& \left.\left(\partial_{t}^{\eta} \mathcal{U}_{t}, \mathcal{U}_{t}\right)_{L_{x}^{2}(\Omega)}-\left(\left(x \mathcal{U}_{x}\right)_{x}, \mathcal{U}_{t}\right)\right)_{L^{2}(\Omega)}+\left(A \mathcal{U}, \mathcal{U}_{t}\right)_{L_{x}^{2}(\Omega)} \\
& \quad=\left(h, \mathcal{U}_{t}\right)_{L_{x}^{2}(\Omega)} .
\end{aligned}
$$

Inequality (5.4) can be reduced to

$$
\begin{aligned}
& 2\left(\partial_{t}^{\eta} \mathcal{U}_{t}, \mathcal{U}_{t}\right)_{L_{x}^{2}(\Omega)}+\frac{\partial}{\partial t}\left\|\mathcal{U}_{x}\right\|_{L_{x}^{2}(\Omega)}^{2}+\frac{\partial}{\partial t}\|\sqrt{A} \mathcal{U}\|_{L_{x}^{2}(\Omega)}^{2} \\
& \quad=2\left(h, \mathcal{U}_{t}\right)_{L_{x}^{2}(\Omega)}+\left\|\sqrt{A_{t}} \mathcal{U}\right\|_{L_{x}^{2}(\Omega)}^{2} .
\end{aligned}
$$

If we integrate (5.5) with respect to $t$ from 0 to $\tau$, and use the Cauchy $\varepsilon$ inequality as well as the conditions (2.4), we obtain

$$
\begin{aligned}
& 2 \cos \left(\frac{\pi \eta}{2}\right)\left\|\partial_{t}^{\eta / 2} \mathcal{U}_{t}\right\|_{L^{2}\left(0, \infty ; L_{x}^{2}(\Omega)\right)}^{2}+\left\|\mathcal{U}_{x}(\cdot \tau)\right\|_{L_{x}^{2}(\Omega)}^{2}+A_{0}\|\mathcal{U}(\cdot, \tau)\|_{L_{x}^{2}(\Omega)}^{2} \\
& \quad \leq \varepsilon\left\|\mathcal{U}_{t}\right\|_{L_{x}^{2}\left(\Lambda_{\tau}\right)}^{2}+\frac{1}{\varepsilon}\|h\|_{L_{x}^{2}\left(\Lambda_{\tau}\right)}^{2}+A_{1}\|\mathcal{U}\|_{L_{x}^{2}\left(\Lambda_{\tau}\right)}^{2} .
\end{aligned}
$$

Thanks to [30], the term $\left\|\mathcal{U}_{t}\right\|_{L_{x}^{2}\left(\Lambda_{\tau}\right)}^{2}$ can be eliminated from the right-hand side of (5.6) and thus

$$
\begin{gathered}
2 \cos \left(\frac{\pi \eta}{2}\right)\left\|\partial_{t}^{\eta / 2} \mathcal{U}_{t}\right\|_{L^{2}\left(0, \infty ; L_{x}^{2}(\Omega)\right)}^{2}+\left\|\mathcal{U}_{x}(\cdot \tau)\right\|_{L_{x}^{2}(\Omega)}^{2}+A_{0}\|\mathcal{U}(\cdot, \tau)\|_{L_{x}^{2}(\Omega)}^{2} \\
\quad \leq \varepsilon \frac{T^{\eta / 2}}{\Gamma\left(\frac{\eta}{2}+1\right)}\left\|\partial_{t}^{\eta / 2} \mathcal{U}_{t}\right\|_{L^{2}\left(0, \tau ; L_{x}^{2}(\Omega)\right)}^{2}+\frac{1}{\varepsilon}\|h\|_{L_{x}^{2}\left(\Lambda_{\tau}\right)}^{2}+A_{1}\|\mathcal{U}\|_{L_{x}^{2}\left(\Lambda_{\tau}\right)^{2}}^{2} .
\end{gathered}
$$

Observe that $\cos \left(\frac{\pi \eta}{2}\right)>0$ and

$$
\left\|\partial_{t}^{\eta / 2} \mathcal{U}_{t}\right\|_{L^{2}\left(0, \infty ; L_{x}^{2}(\Omega)\right)}^{2} \geq\left\|\partial_{t}^{\eta / 2} \mathcal{U}_{t}\right\|_{L^{2}\left(0, \tau ; L_{x}^{2}(\Omega)\right)}^{2},
$$


then letting $\varepsilon=\cos \left(\frac{\pi \eta}{2}\right) \frac{\Gamma\left(\frac{\eta}{2}+1\right)}{T^{\eta / 2}}$, we infer from (5.7) that

$$
\begin{gathered}
\left\|\partial_{t}^{\eta / 2} \mathcal{U}_{t}\right\|_{L^{2}\left(0, \tau ; L_{x}^{2}(\Omega)\right)}^{2}+\|\mathcal{U}(x, \tau)\|_{H_{x}^{1}(\Omega)}^{2} \\
\leq \theta\left(\|h\|_{L_{x}^{2}\left(\Lambda_{\tau}\right)}^{2}+\|\mathcal{U}\|_{H_{x}^{1}\left(\Lambda_{\tau}\right)}^{2}\right)
\end{gathered}
$$

where

$$
\theta=\frac{\max \left(\frac{T^{\eta / 2}}{\cos \left(\frac{\pi \eta}{2}\right) \cdot \Gamma\left(\frac{\eta}{2}+1\right)}, A_{1}\right)}{\min \left(\cos \left(\frac{\pi \eta}{2}\right), 1\right)} .
$$

A term $\|\mathcal{U}\|_{L_{x}^{2}\left(\Lambda_{\tau}\right)}^{2}$ was added to the right-hand side of (5.7). We now apply Gronwall's lemma [11] to (5.9), we obtain after taking the maximum over the interval $[0, T]$

$$
\max _{0 \leq \tau \leq T}\|\mathcal{U}(x, \tau)\|_{\left.H_{x}^{1}(\Omega)\right)}^{2}+\left\|\partial_{t}^{\eta / 2} \mathcal{U}_{t}\right\|_{L_{x}^{2}(\Lambda)}^{2} \leq \theta \exp (\theta T)\|h\|_{L_{x}^{2}(\Lambda)}^{2}
$$

from which we deduce the uniqueness and continuous dependence of solution on the input data of problem (5.1), (2.2) and (2.3).

Theorem 5.2 Let $\lambda=\eta+1$ with $0<\eta<1$, and assume that $h \in L_{x}^{2}([0, T],(0, b))$, then the solution of problem (5.1), (2.2) and (2.3) satisfies the a priori estimate

$$
\begin{aligned}
& \max _{0 \leq t \leq T}\left\|\partial_{t}^{\eta} \mathcal{U}(x, t)\right\|_{L_{x}^{2}(\Omega)}+\left\|\partial_{t}^{\eta / 2} \mathcal{U}\right\|_{H_{x}^{1}(\Lambda)} \\
& \quad \leq d\|h\|_{L_{x}^{2}(\Lambda)}
\end{aligned}
$$

where

$$
d=2 \sqrt{T}\left(1+\frac{1}{\sqrt{\min \left(\cos (\pi \eta / 2), A_{0} \cos (\pi \eta / 2)\right)}}\right) .
$$

Proof We multiply the differential equation (5.1) by $x \partial_{t}^{\eta} \mathcal{U}$ and integrate on $\Omega$, we have

$$
\begin{aligned}
& \left(\partial_{t}^{\eta+1} \mathcal{U}, \partial_{t}^{\eta} \mathcal{U}\right)_{L_{x}^{2}(\Omega)}-\left(\left(x \mathcal{U}_{x}\right)_{x}, \partial_{t}^{\eta} \mathcal{U}\right)_{L^{2}(\Omega)}+\left(A \mathcal{U}, \partial_{t}^{\eta} \mathcal{U}\right)_{L_{x}^{2}(\Omega)} \\
& \quad=\left(h, \partial_{t}^{\eta} \mathcal{U}\right)_{L_{x}^{2}(\Omega)}
\end{aligned}
$$

The use of initial and boundary conditions gives

$$
\begin{aligned}
& \frac{1}{2} \frac{\partial}{\partial t}\left\|\partial_{t}^{\eta} \mathcal{U}\right\|_{L_{x}^{2}(\Omega)}^{2}+\left(\partial_{t}^{\eta} \mathcal{U}_{x}, \mathcal{U}_{x}\right)_{L_{x}^{2}(\Omega)}+\left(A \mathcal{U}, \partial_{t}^{\eta} \mathcal{U}\right)_{L_{x}^{2}(\Omega)} \\
& \quad=\left(h, \partial_{t}^{\eta} \mathcal{U}\right)_{L_{x}^{2}(\Omega)}
\end{aligned}
$$


Invoking conditions (2.4) and replacing $t$ by $v$ and integrating with respect to $v$ from 0 to $t$, inequality (5.14) implies

$$
\begin{gathered}
\left\|\partial_{t}^{\eta} \mathcal{U}(x, t)\right\|_{L_{x}^{2}(\Omega)}^{2}+2 \cos \left(\frac{\pi \eta}{2}\right)\left\|\partial_{t}^{\eta / 2} \mathcal{U}_{x}\right\|_{L^{2}\left(0, \infty ; L_{x}^{2}(\Omega)\right)}^{2} \\
+A_{0} \cos \left(\frac{\pi \eta}{2}\right)\left\|\partial_{t}^{\eta / 2} \mathcal{U}\right\|_{L^{2}\left(0, \infty ; L_{x}^{2}(\Omega)\right)}^{2} \\
=2\left(h, \partial_{t}^{\eta} \mathcal{U}\right)_{L^{2}\left(0, t ; L_{x}^{2}(\Omega)\right)}+\left\|\partial_{t}^{\eta} \mathcal{U}(x, 0)\right\|_{L_{x}^{2}(\Omega)}^{2} .
\end{gathered}
$$

Taking into account that

$$
\left\{\begin{array}{l}
\left\|\partial_{t}^{\eta / 2} \mathcal{U}_{x}\right\|_{L^{2}\left(0, \infty ; L_{x}^{2}(\Omega)\right)}^{2} \geq\left\|\partial_{t}^{\eta / 2} \mathcal{U}_{x}\right\|_{L_{x}^{2}\left(\Lambda_{t}\right)}^{2}, \\
\left\|\partial_{t}^{\eta / 2} \mathcal{U}\right\|_{L^{2}\left(0, \infty ; L_{x}^{2}(\Omega)\right)}^{2} \geq\left\|\partial_{t}^{\eta / 2} \mathcal{U}\right\|_{L_{x}^{2}\left(\Lambda_{t}\right)^{\prime}}^{2}
\end{array}\right.
$$

and employing condition (2.2) and the Cauchy-Schwartz inequality, we infer from (5.15) that

$$
\begin{aligned}
2 \cos & \left(\frac{\pi \eta}{2}\right)\left\|\partial_{t}^{\eta / 2} \mathcal{U}_{x}\right\|_{L_{x}^{2}\left(\Lambda_{t}\right)}^{2}+A_{0} \cos \left(\frac{\pi \eta}{2}\right)\left\|\partial_{t}^{\eta / 2} \mathcal{U}\right\|_{L_{x}^{2}\left(\Lambda_{t}\right)}^{2} \\
& +\left\|\partial_{t}^{\eta} \mathcal{U}(x, t)\right\|_{L_{x}^{2}(\Omega)}^{2} \\
\leq & 2 \sqrt{T} \max _{0 \leq t \leq T}\left\|\partial_{t}^{\eta} \mathcal{U}(x, t)\right\|_{L_{x}^{2}(\Omega)}\|h\|_{L_{x}^{2}\left(\Lambda_{t}\right)} .
\end{aligned}
$$

Discarding the first two term on the left-hand side of (5.17) gives

$$
\max _{0 \leq t \leq T}\left\|\partial_{t}^{\eta} \mathcal{U}(x, t)\right\|_{L_{x}^{2}(\Omega)} \leq 2 \sqrt{T}\|h\|_{L_{x}^{2}(\Lambda)}
$$

Now dropping the third term on the left-hand side of (5.17), then using (5.18), and taking $t=T$, we obtain

$$
\left\|\partial_{t}^{\eta / 2} \mathcal{U}\right\|_{H_{x}^{1}(\Lambda)} \leq 2 \sqrt{T}\|h\|_{L_{x}^{2}(\Lambda)} \frac{1}{\sqrt{\min \left(\cos (\pi \eta / 2), A_{0} \cos (\pi \eta / 2)\right)}} .
$$

By summing (5.18) and (5.19) side to side, the a priori estimate (5.11) follows, and it implies that problem (5.1), (2.2) and (2.3) admits a unique solution depending continuously on the given data.

Conclusion 1 A collection of a priori estimates in fractional Sobolev spaces for the solution of some initial boundary value problems for singular time-fractional diffusion equations with mass absorption is obtained. The fractional derivative is considered in the Riemann-Liouville sense. For this type of fractional derivatives, an arbitrary function needs not be continuous at the origin and it needs not be differentiable. The uniqueness and continuous dependence (stability of solution) of the solution on the input data in different fractional Sobolev spaces follows from the obtained a priori bounds.

The method of energy inequalities is used to obtain the different a priori estimates. The obtained results can greatly contribute in the development of the used method for partial 
differential equations of fractional order. We should mention that there are few papers using the a priori estimate method for the fractional order differential equations. A more general fractional models can be investigated by employing the proposed method, especially those having a certain singularity, such as fractional evolution equations with Bessel operator, where weighted Sobolev spaces may be involved.

\section{Acknowledgements}

The authors would like to extend their sincere appreciation to the Deanship of Scientific Research at King Saud University for its funding this Research group No. (RG-117).

\section{Funding}

The Deanship of Scientific Research at King Saud University.

Availability of data and materials

Not applicable.

\section{Competing interests}

The authors declare that they have no competing interests.

\section{Authors' contributions}

The authors read and approved the final manuscript.

\section{Publisher's Note}

Springer Nature remains neutral with regard to jurisdictional claims in published maps and institutional affiliations.

Received: 27 July 2020 Accepted: 11 October 2020 Published online: 17 October 2020

\section{References}

1. Blackledge, J., Babajanov, B.: The fractional Schrödinger-Klein-Gordon equation and intermediate relativism. Math. Æterna 3,601-615 (2013)

2. Vitali, S., Castellani, G., Mainardi, F.: Time fractional cable equation and applications in neurophysiology. Chaos Solitons Fractals 102, 467-472 (2017)

3. Povstenko, Y., Kyrylych, T.: Time-fractional diffusion with mass absorption under harmonic impact. Fract. Calc. Appl. Anal. 21, 118-133 (2018). https://doi.org/10.1515/fca-2018-0008

4. Povstenko, Y., Kyrylych, T.: Time-fractional diffusion with mass absorption in a half-line domain due to boundary value of concentration varying harmonically in time. Entropy 19, 346 (2018)

5. Jordan, P.M., Puri, A.: Digital signal propagation in dispersive media. J. Appl. Phys. 85, 1273-1282 (1999)

6. Weston, V.H., He, S.: Wave splitting of the telegraph equation in $\mathrm{R}^{3}$ and its application to inverse scattering. Inverse Probl. 9, 789-812 (1993)

7. Hilfer, R. (ed.): Applications of Fractional Calculus in Physics World Scientific, Singapore (2000)

8. Mainardi, F.: Fractional calculus: some basic problems in continuum and statistical mechanics. In: Carpinteri, A., Mainardi, F. (eds.) Fractals and Fractional Calculus in Continuum Mechanics, pp. 291-348. Springer, Wien (1997)

9. Zaslavsky, G.M.: Chaos, fractional kinetics, and anomalous transport. Phys. Rep. 371, 461-580 (2002)

10. Srivastava, H.M., Jena, R.M., Chakraverty, S., et al.: Dynamic response analysis of fractionally-damped generalized Bagley-Torvik equation subject to external loads. Russ. J. Math. Phys. 27, 254-268 (2020)

11. Srivastava, H.M., Shah, F.A., Abass, R.: An application of the Gegenbauer wavelet method for the numerical solution of the fractional Bagley-Torvik equation. Russ. J. Math. Phys. 26, 77-93 (2019)

12. Baleanu, D., Shiri, B., Srivastava, H.M., Al Qurashi, M.: A Chebyshev spectral method based on operational matrix for fractional differential equations involving non-singular Mittag-Leffler kernel. Adv. Differ. Equ. 2018, Article ID 353 (2018)

13. Singh, H., Srivastava, H.M.: Jacobi collocation method for the approximate solution of some fractional-order Riccati differential equations with variable coefficients. Phys. A, Stat. Mech. Appl. 523(C), 1130-1149 (2019)

14. Shiri, B., Wu, G.C., Baleanu, D.: Collocation methods for terminal value problems of tempered fractional differential equations. Appl. Numer. Math. 156, 385-395 (2020)

15. Chang, Y.M., Shiri, B., Wu, G.C., Baleanu, D.: New fractional signal smoothing equations with short memory and variable order. Optik 218, 164507 (2020)

16. Ehsan, D.K., Hosain, G., Shiri, B.: Spline collocation methods for seismic analysis of multiple degree of freedom systems with visco-elastic dampers using fractional models. J. Vib. Control 26(17-18), 1445-1462 (2020)

17. Shiri, B., Baleanu, D.: System of fractional differential algebraic equations with applications. Chaos Solitons Fractals 120, 203-212 (2019)

18. Baleanu, D., Shiri, B.: Collocation methods for fractional differential equations involving non-singular kernel. Chaos Solitons Fractals 116, 136-145 (2018)

19. Singh, H., Srivastava, H.M., Kumar, D.: A reliable numerical algorithm for the fractional vibration equation. Chaos Solitons Fractals 103, 131-138 (2017)

20. Singh, H., Srivastava, H.M.: Numerical simulation for fractional-order Bloch equation arising in nuclear magnetic resonance by using the Jacobi polynomials. Appl. Sci. 10, 2850 (2020). https://doi.org/10.3390/app10082850 
21. Singh, H., Srivastava, H.M.: Numerical investigation of the fractional order Liénard and Duffing equations arising in oscillating circuit theory. Front. Phys. 8, 120 (2020)

22. Kasmi, L., Guerfi, A., Mesloub, S.: Existence of solution for 2-D time-fractional differential equations with a boundary integral condition. Adv. Differ. Equ. 2019, Article ID 511 (2019)

23. Mesloub, S., Bachar, I.: On a nonlocal 1-D initial value problem for a singular fractional-order parabolic equation with Bessel operator. Adv. Differ. Equ. 2019, Article ID 254 (2019)

24. Alikhanov, A.A.: A priori estimates for solutions of boundary value problems for fractional-order equations. Differ. Equ. 46(5), 660-666 (2010)

25. Podlubny, I.: Fractional Differential Equations. Academic Press, New York (1999)

26. Kilbas, A.A., Srivastava, H.M., Trujillo, J.J.: Theory and Applications of Fractional Differential Equations. Elsevier, Amsterdam (2006)

27. Samko, S.G., Kilbas, A.A., Marichev, O.I.: Fractional Integrals and Derivatives, Theory and Applications. Gordon and Breach, Amsterdam (1993). English translation from Russian edition: Naukai Tekhnika, Minsk (1987)

28. Miller, K.S., Ross, B.: An Introduction to the Fractional Calculus and Fractional Differential Equations. Wiley, New York (1993)

29. Delic, A.: Fractional in time diffusion-wave equation and its numerical approximation. Filomat 30(5), 1375-1385 (2016)

30. Ervin, V.J., Roop, J.P.: Variational formulation for the stationary fractional advection dispersion equation. Numer. Methods Partial Differ. Equ. 23, 558-576 (2006)

Submit your manuscript to a SpringerOpen ${ }^{\circ}$ journal and benefit from:

- Convenient online submission

- Rigorous peer review

- Open access: articles freely available online

- High visibility within the field

- Retaining the copyright to your article

Submit your next manuscript at $\boldsymbol{~ s p r i n g e r o p e n . c o m ~}$ 